\title{
DOI: 10.33947/1982-3282-v15n3-4-4745 \\ ADESIVO UNIVERSAL NO TRATAMENTO RESTAURADOR DE LESÕES CERVICAIS NÃO CARIOSAS: ESTRATÉGIAS DE ADESÃO NA SENSIBILIDADE PÓS-OPERATÓRIA
}

\author{
UNIVERSAL ADHESIVE IN THE RESTORATIVE TREATMENT OF NON-CARIOUS CERVICAL INJURIES: \\ ADHESION STRATEGIES IN POST-OPERATIVE SENSITIVITY
}

\section{ADHESIVO UNIVERSAL EN EL TRATAMIENTO RESTAURATIVO DE LESIONES CERVICALES NO CARIOS: ESTRATEGIAS DE ADHESIÓN EN SENSIBILIDAD POSTOPERATORIA}

\author{
André Felipe Waz ${ }^{1}$, Cristiane Franco Pinto²
}

Submetido em: $27 / 08 / 2021$

Aceito em: 28/09/2021

\section{RESUMO}

Introdução: A hipersensibilidade dentinária é um quadro comum em pacientes com lesões cervicais não cariosas. Objetivo: Analisar o grau de sensibilidade pós-operatória no tratamento restaurador de lesões cervicais não cariosas utilizando um sistema adesivo universal em diferentes estratégias de adesão. Método: Quinze voluntários com lesões cervicais não cariosas foram submetidos a procedimentos restauradores com o sistema adesivo Ambar Universal. Os voluntários foram divididos em dois grupos experimentais: 1) substrato seco utilizando a Técnica Autocondicionante e 2) substrato úmido utilizando a Técnica Condicione e Lave. Foram realizadas as mensurações da sensibilidade antes e após sete dias do tratamento restaurador por meio de estímulos táteis e evaporativos. Resultados: A sensibilidade no pós-operatório de sete dias diminuiu significativamente em ambos os grupos após o tratamento restaurador. Contudo, não houve diferenças nos escores de sensibilidade no pós-operatório comparando os dois grupos. Conclusão: Em ambas as estratégias de adesão o adesivo Ambar Universal produziu resultados semelhantes em relação à sensibilidade pós-operatória de sete dias.

DESCRITORES: Hipersensibilidade Dentinária; Sistemas Adesivos Universais; Lesões Cervicais Não Cariosas.

\section{ABSTRACT}

Introduction: Dentin hypersensitivity is a common condition in patients with non-carious cervical lesions. Objective: To analyze the degree of postoperative sensitivity in the restorative treatment of non-carious cervical lesions using a universal adhesive system in different adhesion strategies. Method: Fifteen volunteers with non-carious cervical lesions underwent restorative procedures with the Ambar Universal adhesive system. The volunteers were divided into two experimental groups: 1) dry substrate using the Self-Etching Technique and 2) wet substrate using the Condition and Wash Technique. Sensitivity measurements were performed before and after seven days of restorative treatment through tactile and evaporative stimuli. Results: The seven-day postoperative sensitivity significantly decreased in both groups after restorative treatment. However, there were no differences in postoperative sensitivity scores comparing the two groups. Conclusion: In both adhesion strategies, the Ambar Universal adhesive produced similar results regarding the seven-day postoperative sensitivity.

DESCRIPTORS: Dentin Hypersensitivity; Universal Adhesive Systems; Non-carious Cervical Lesions.

\footnotetext{
${ }^{1}$ Graduado em Odontologia. Universidade São Francisco - USF. https://orcid.org/0000-0002-5479-1237. E-mail: felipe.waz@ hotmail.com - Fone: +55 1199690 0024. Endereço: Rua Suíça, número 130, Jardins, Bragança Paulista, São Paulo, Brasil. ${ }^{2}$ Mestre e Doutora em Clínica Odontológica na área de Dentística. Universidade São Francisco - USF. https://orcid.org/00000002-5638-4539. E-mail: crisfp2009@hotmail.com.
} 


\section{RESUMEN}

Introducción: La hipersensibilidad dentinaria es una condición común en pacientes con lesiones cervicales no cariosas. Objetivo: Analizar el grado de sensibilidad postoperatoria en el tratamiento restaurador de lesiones cervicales no cariosas utilizando un sistema adhesivo universal en diferentes estrategias de adhesión. Método: Quince voluntarios con lesiones cervicales no cariosas se sometieron a procedimientos de restauración con el sistema adhesivo Ambar Universal. Los voluntarios se dividieron en dos grupos experimentales: 1) sustrato seco usando la técnica de autograbado y 2) sustrato húmedo usando la técnica de acondicionamiento y lavado. Las mediciones de sensibilidad se realizaron antes y después de siete días de tratamiento restaurador mediante estímulos táctiles y evaporativos. Resultados: La sensibilidad posoperatoria de siete días disminuyó significativamente en ambos grupos después del tratamiento restaurador. Sin embargo, no hubo diferencias en las puntuaciones de sensibilidad posoperatoria al comparar los dos grupos. Conclusión: En ambas estrategias de adhesión, el adhesivo Ambar Universal produjo resultados similares en cuanto a la sensibilidad posoperatoria a los siete días.

DESCRIPTORES: Hipersensibilidad dentinaria; Sistemas adhesivos universales; Lesiones cervicales no cariosas. 


\section{INTRODUÇÃO}

As lesões cervicais não cariosas são definidas como uma perda de estrutura dentária resultante de uma etiologia variável que não envolve microrganismos, mas hábitos de higiene, parafuncionais e de dieta, sendo classificadas em abrasão, abfração e erosão, e podem aparecer de formas isoladas ou associadas a outros fatores, como a hipersensibilidade dentinária e a recessão gengival. Deste modo, é imprescindível conhecer cada fator etiológico que de alguma forma estava relacionado ao desenvolvimento da lesão para esclarecer sua causa, diagnóstico, tratamento e prevenção1,2. A prevalência mundial de lesões cervicais não cariosas entre adultos é de 46,7\%, mais comum em indivíduos idosos do que em jovens3.

A hipersensibilidade dentinária é caracterizada como uma dor aguda e passageira que surge devido à exposição coronal ou radicular da dentina. Segundo Brännström, o mecanismo da dor após a dentina exposta pode ser explicado através da "Teoria da Hidrodinâmica", onde a movimentação desses fluídos pelos túbulos dentinários abertos no meio bucal estimulam odontoblastos, que ativam suas terminações nervosas, provocando assim algum tipo de sensibilidade frente a estímulos térmicos, táteis, osmóticos, químicos ou evaporativos4,5,6. Desse modo, uma modalidade de tratamento bem-sucedida deve ser capaz de aliviar os sintomas impedindo a transmissão neural dos nervos pulpares ou ocluindo os túbulos da dentina, bloqueando o mecanismo hidrodinâmico7.

Em 1955, Buonocore criou uma nova perspectiva nos procedimentos restauradores dando início à odontologia adesiva. Nessa ocasião, os avanços científicos e tecnológicos sobre os adesivos dentários influenciaram e revolucionaram extensivamente muitos aspectos da Odontologia Restauradora 8, 9, 10. As restaurações adesivas aderem à estrutura dentária por meio dos sistemas adesivos, que são soluções de monômeros de resina em um solvente orgânico com iniciadores de polimerização. O sucesso de restaurações adesivas depende, portanto, de obter uma forte ligação com os substratos através das estratégias de adesão que são agrupadas em duas categorias básicas: 1) Técnica Condicione e Lave e 2) Técnica Autocondicionante 8, 9, 11.

Assim, os sistemas adesivos universais, ou "multimodais", recém-introduzidos no mercado odontológico rapidamente chamaram a atenção dos cirurgiões dentistas devido seu modo de aplicação flexível, considerando que em sua composição todos os ingredientes foram incorporados em um único frasco 12. Esses sistemas são mais práticos porque podem ser aplicados em ambas as estratégias de adesão 13, oferecendo assim uma versatilidade de uso, pois se ligam adequadamente à estrutura dentária e a diferentes materiais restauradores 9 .

Portanto, o objetivo deste estudo foi analisar a diferença no grau de sensibilidade pós-operatória no tratamento restaurador de lesões cervicais não cariosas utilizando um sistema adesivo universal em diferentes técnicas de adesão no substrato dental.

\section{MÉTODO}

\section{SELEÇÃO DOS VOLUNTÁRIOS}

Este projeto foi aprovado pelo Comitê de Ética em Pesquisa da Universidade São Francisco (CAAE 19960919.4.0000.5514). A amostra experimental foi selecionada dentre os pacientes da Clínica de Odontologia da Universidade São Francisco e todos os voluntários assinaram o Termo de Consentimento Livre e Esclarecido.

Os critérios de inclusão dos voluntários foram: 1) duas lesões cervicais não cariosas com exposição do tecido dentinário ao meio bucal em pré-molares, sendo uma lesão em uma hemiarcada e outra lesão na hemiarcada oposta, 2) demonstrar dor ou desconforto ao estímulo tátil e/ou evaporativo; 3) não ter utilizado flúor tópico ou dessensibilizantes nos últimos 3 meses. Os critérios de exclusão foram pacientes que estavam sob tratamento com antibióticos, analgésicos ou anti-inflamatórios.

Quinze voluntários participaram deste estudo, no qual cada voluntário participou dos dois grupos experimentais: 1) substrato seco utilizando a Técnica Autocondicionante e 2) substrato úmido utilizando a Técnica Condicione e Lave. Sendo assim, cada paciente foi submetido em ambas às estratégias adesivas. 


\section{PROTOCOLO DE TRATAMENTO}

O tratamento restaurador foi realizado em uma consulta, inicialmente foi feito uma profilaxia na região a ser restaurada com uma taça de borracha e pasta profilática. Em seguida foi executada a lavagem com jato de ar e água, secagem da cavidade e seleção da cor da resina composta a ser utilizada. Foi posicionado o afastador labial, realizado um isolamento relativo do campo operatório com roletes de algodão e inserido o fio retrator no sulco gengival. Todos os procedimentos foram realizados com o adesivo Ambar Universal (FGM) e a Resina Composta Opallis (FGM), a aplicação dos materiais foi padronizada conforme determina os fabricantes.

No Grupo 1 o sistema adesivo Ambar Universal foi aplicado em duas camadas. Cada camada foi aplicada através de um microaplicador embebido do produto esfregando durante 20 segundos e volatilizado com jatos de ar por 5 segundos. Em seguida, foi realizado a fotopolimeração com luz azul por 20 segundos. Logo após, as lesões foram restauradas com a resina composta Opallis através da técnica de inserção e polimerização por pequenos incrementos, a cada incremento foi realizado a fotopolimeração com luz azul por 20 segundos.

As lesões do Grupo 2 foram condicionadas com ácido fosfórico $35 \%$ por 30 segundos em esmalte e 15 segundos em dentina, lavadas com jatos de ar e água por 1 minuto, secadas com bolinha de algodão na dentina e jatos de ar no esmalte. O sistema adesivo Ambar Universal foi aplicado em duas camadas. Cada camada foi aplicada através de um microaplicador embebido do produto esfregando durante 20 segundos e volatilizado com jatos de ar por 5 segundos. Em seguida, foi realizado a fotopolimeração com luz azul por 20 segundos. Logo após, as lesões foram restauradas com a resina composta Opallis, conforme descrito no Grupo 1.

Após o procedimento restaurador, foi realizado o acabamento com pontas diamantadas $\mathrm{F}$ e FF para remoção dos excessos de resina composta e adesivo. O polimento foi realizado após sete dias com pontas de silicone e pasta diamantada.

\section{ANÁLISE DE SENSIBILIDADE}

Cada voluntário recebeu as instruções verbais e padronizadas quanto à manipulação da escala visual analógica de dor (EVA), que consiste em um retângulo de $10,0 \mathrm{~cm}$ de comprimento, com a parte da frente marcada pelas descrições nas extremidades "nenhuma dor" e "dor insuportável" e, na parte de trás, uma régua milimetrada de 10,0cm acoplada à escala, sendo esta régua não visível pelo paciente. Inicialmente, antes do procedimento restaurador, cada paciente recebeu quatro estímulos táteis com o atrito leve de uma sonda exploradora, ocorrendo um intervalo de dois minutos entre cada estímulo (estímulos 1, 2, 3 e 4 ), estes testes foram realizados no terço médio e no terço cervical da face vestibular dos dentes. Após dez minutos da aplicação dos estímulos táteis, foram realizados quatro estímulos evaporativos nos mesmos dentes, ocorrendo um intervalo de dois minutos entre cada estímulo (estímulos 5, 6, 7 e 8), consistindo na aplicação do ar da seringa tríplice por 3 segundos, direcionada perpendicularmente à superfície dental e afastada $2,0 \mathrm{~mm}$ do mesmo. O voluntário puxou a parte móvel da escala de acordo com a intensidade do desconforto experimentado e o avaliador registrou o número correspondente a parte de trás da escala. Sete dias após o tratamento restaurador, os voluntários tiveram um acompanhamento do pós-operatório no qual as mensurações da sensibilidade dental foram realizadas novamente no mesmo padrão já realizado anteriormente.

\section{ANÁLISE ESTATÍSTICA}

Os dados foram tabulados e analisados estatisticamente no SPSS versão 20.0 (IBM Corp., Armonk, NY, EUA). O teste não-paramétrico de Wilcoxon foi aplicado para comparar os dados de amostras pareadas e o teste não-paramétrico de Mann-Whitney foi aplicado para comparar os dados de amostras independentes, ambas com nível de significância $5 \%(P<0,05)$.

\section{RESULTADOS}

No total, 15 pacientes foram incluídos neste estudo sendo 30 dentes avaliados. Nas tabelas 1 e 2 apontam DOI: $10.33947 / 1982-3282-v 15 n 3-4-4745$ 
que a sensibilidade diminuiu estatisticamente em ambos os grupos $(p<0,05)$ no pós-operatório de sete dias do tratamento restaurador.

Tabela 1. Valores estatísticos do Grupo 1 referente ao grau de sensibilidade antes e depois de sete dias do tratamento restaurador, entre duas amostras pareadas utilizando o teste de Wilcoxon.

\begin{tabular}{|c|c|c|c|c|c|c|c|}
\hline \multicolumn{8}{|c|}{ G1 - Substrato Seco } \\
\hline Testes de Sensibilidade & $\mathbf{N}$ & Média & $\begin{array}{l}\text { Desvio } \\
\text { Padrão }\end{array}$ & Mínimo & Máximo & Mediana & $\begin{array}{c}\text { Valor } \\
\mathbf{P}\end{array}$ \\
\hline \multicolumn{8}{|l|}{ Teste Tátil Terço Médio } \\
\hline Antes & 15 & 0,07 & 0,258 & 0 & 1 & 0,00 & \multirow{2}{*}{0,317} \\
\hline Depois & 15 & 0,00 & 0,000 & 0 & 0 & 0,00 & \\
\hline \multicolumn{8}{|l|}{ Teste Tátil Terço Cervical } \\
\hline Antes & 15 & 1,80 & 1,265 & 0 & 4 & 1,00 & \multirow{2}{*}{$0,001^{*}$} \\
\hline Depois & 15 & 0,00 & 0,000 & 0 & 0 & 0,00 & \\
\hline \multicolumn{8}{|l|}{ Teste Evaporativo Terço Médio } \\
\hline Antes & 15 & 1,93 & 1,668 & 0 & 5 & 2,00 & \multirow{2}{*}{$0,002^{*}$} \\
\hline Depois & 15 & 0,07 & 0,258 & 0 & 1 & 0,00 & \\
\hline \multicolumn{8}{|l|}{ Teste Evaporativo Terço } \\
\hline \multicolumn{8}{|l|}{ Cervical } \\
\hline Antes & 15 & 4,80 & 1,699 & 3 & 8 & 4,00 & \multirow{2}{*}{$0,001^{*}$} \\
\hline Depois & 15 & 0,20 & 0,775 & 0 & 3 & 0,00 & \\
\hline
\end{tabular}

Fonte: Dados da pesquisa, 2021.

Tabela 2. Valores estatísticos do Grupo 2 referente ao grau de sensibilidade antes e depois de sete dias do tratamento restaurador, entre duas amostras pareadas utilizando o teste de Wilcoxon.

\begin{tabular}{|c|c|c|c|c|c|c|c|}
\hline \multicolumn{8}{|c|}{ G2 - Substrato Úmido } \\
\hline Testes de Sensibilidade & $\mathbf{N}$ & Média & $\begin{array}{l}\text { Desvio } \\
\text { Padrão }\end{array}$ & Mínimo & Máximo & Mediana & $\begin{array}{c}\text { Valor } \\
\mathbf{P}\end{array}$ \\
\hline \multicolumn{8}{|l|}{ Teste Tátil Terço Médio } \\
\hline Antes & 15 & 0,00 & 0,000 & 0 & 0 & 0,00 & \multirow{2}{*}{1,000} \\
\hline Depois & 15 & 0,00 & 0,000 & 0 & 0 & 0,00 & \\
\hline \multicolumn{8}{|l|}{ Teste Tátil Terço Cervical } \\
\hline Antes & 15 & 1,27 & 0,704 & 0 & 3 & 1,00 & \multirow{2}{*}{$0,001^{*}$} \\
\hline Depois & 15 & 0,00 & 0,000 & 0 & 0 & 0,00 & \\
\hline \multicolumn{8}{|l|}{ Teste Evaporativo Terço Médio } \\
\hline Antes & 15 & 1,93 & 1,624 & 0 & 5 & 2,00 & \multirow{2}{*}{$0,005^{\star}$} \\
\hline Depois & 15 & 0,27 & 0,594 & 0 & 2 & 0,00 & \\
\hline \multicolumn{8}{|l|}{ Teste Evaporativo Terço } \\
\hline \multicolumn{8}{|l|}{ Cervical } \\
\hline Antes & 15 & 4,47 & 1,552 & 3 & 7 & 4,00 & \multirow{2}{*}{$0,001^{*}$} \\
\hline Depois & 15 & 0,87 & 1,302 & 0 & 3 & 0,00 & \\
\hline
\end{tabular}

Fonte: Dados da pesquisa, 2021. 
Na tabela 3 aponta que não houve diferenças estatísticas comparando o grau de sensibilidade antes do tratamento restaurador entre os Grupos 1 e $2(p>0,05)$, ou seja, o nível de sensibilidade previamente o tratamento era igual em ambos os grupos.

No gráfico 1 apresenta que o grupo Substrato Seco obteve um menor grau de sensibilidade no pós-operatório de sete dias do tratamento restaurador. No entanto, não houve diferenças estatísticas significativas nos escores de sensibilidade no pós-operatório de sete dias comparando os dois grupos $(p>0,05)$ como demonstra na tabela 4 .

Tabela 3. Valores estatísticos dos Grupos 1 e 2, em análise do grau de sensibilidade antes do tratamento restaurador, entre duas amostras independentes utilizando o teste de Mann-Whitney.

\begin{tabular}{lccccccccc}
\hline \multicolumn{8}{c}{ Comparação entre G1 - Substrato Seco e G2 - Substrato Úmido } \\
\hline $\begin{array}{l}\text { Testes de Sensibilidade } \\
\begin{array}{c}\text { Antes do Tratamento } \\
\text { Restaurador }\end{array}\end{array}$ & N & Média & $\begin{array}{c}\text { Desvio } \\
\text { Padrão }\end{array}$ & Mínimo & Máximo & Mediana & $\begin{array}{c}\text { Mhitney } \\
\text { U }\end{array}$ & $\begin{array}{c}\text { Valor } \\
\text { P }\end{array}$ \\
\hline Teste Tátil Terço Médio & 30 & 0,03 & 0,183 & 0 & 1 & 0,00 & 105,000 & 0,317 \\
\hline Teste Tátil Terço Cervical & 30 & 1,53 & 1,042 & 0 & 4 & 1,00 & 85,000 & 0,219 \\
\hline $\begin{array}{l}\text { Teste Evaporativo Terço } \\
\text { Médio }\end{array}$ & 30 & 1,93 & 1,617 & 0 & 5 & 2,00 & 111,000 & 0,949 \\
\hline $\begin{array}{l}\text { Teste Evaporativo Terço } \\
\text { Cervical }\end{array}$ & 30 & 4,63 & 1,608 & 3 & 8 & 4,00 & 99,500 & 0,578 \\
\hline
\end{tabular}

Fonte: Dados da pesquisa, 2021.

Gráfico 1. Análise dos Grupos 1 e 2, em relação ao grau de sensibilidade antes e depois de sete dias do tratamento restaurador.

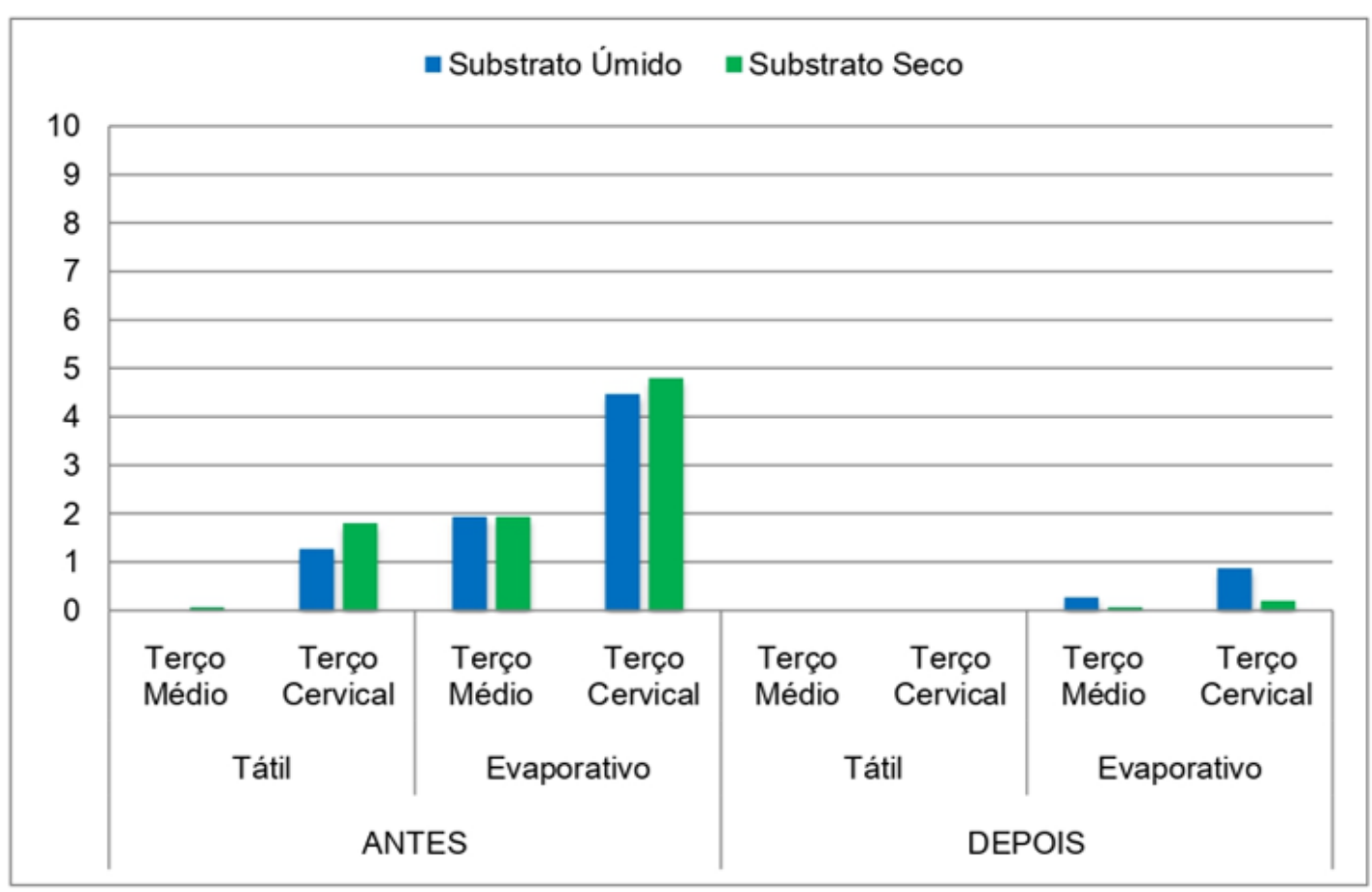

Fonte: Dados da pesquisa, 2021. 
Tabela 4. Valores estatísticos dos Grupos 1 e 2, em análise do grau de sensibilidade depois do tratamento restaurador, entre duas amostras independentes utilizando o teste de Mann-Whitney.

\begin{tabular}{|c|c|c|c|c|c|c|c|c|}
\hline \multicolumn{9}{|c|}{ Comparação entre G1 - Substrato Seco e G2 - Substrato Úmido } \\
\hline $\begin{array}{c}\text { Testes de Sensibilidade } \\
\text { Depois do Tratamento } \\
\text { Restaurador }\end{array}$ & $\mathbf{N}$ & Média & $\begin{array}{l}\text { Desvio } \\
\text { Padrão }\end{array}$ & Mínimo & Máximo & Mediana & $\begin{array}{c}\text { Mann } \\
\text { Whitney } \\
\text { U }\end{array}$ & $\begin{array}{c}\text { Valor } \\
\mathbf{P}\end{array}$ \\
\hline Teste Tátil Terço Médio & 30 & 0,00 & 0,000 & 0 & 0 & 0,00 & 112,500 & 1,000 \\
\hline Teste Tátil Terço Cervical & 30 & 0,00 & 0,000 & 0 & 0 & 0,00 & 112,500 & 1,000 \\
\hline $\begin{array}{l}\text { Teste Evaporativo Terço } \\
\text { Médio }\end{array}$ & 30 & 0,17 & 0,461 & 0 & 2 & 0,00 & 97,000 & 0,276 \\
\hline $\begin{array}{l}\text { Teste Evaporativo Terço } \\
\text { Cervical }\end{array}$ & 30 & 0,53 & 1,106 & 0 & 3 & 0,00 & 83,500 & 0,084 \\
\hline
\end{tabular}

Fonte: Dados da pesquisa, 2021.

\section{DISCUSSÃO}

O objetivo deste estudo foi analisar o grau de sensibilidade pós-operatória no tratamento restaurador de lesões cervicais não cariosas utilizando o adesivo Ambar universal em diferentes estratégias de adesão no substrato dental. A hipótese nula testada foi aceita, pois não houve diferença estatística significativa entre os dois grupos quanto à sensibilidade pós-operatória de sete dias do tratamento.

As lesões cervicais não cariosas são causadas pela perda da estrutura dentária resultante de uma etiologia variável que não envolve microrganismos, que podem aparecer de forma isolada ou associada a outros fatores, como a hipersensibilidade dentinária e a recessão gengival 1, 2, 14. Assim, cada tipo de lesão cervical não cariosa tem suas características clínicas próprias e condutas terapêuticas diferentes a serem associados ao tratamento da hipersensibilidade. Encontra-se, na literatura, uma vasta variedade de tratamentos, porém ainda não há um protocolo padrão estipulado 15,16 . No presente estudo, o tratamento restaurador foi optado visto ser um procedimento adequado, eficiente e duradouro quanto à redução da hipersensibilidade dentinária, principalmente quando a perda estrutural e a condição estética estão comprometidas, tendo como objetivo principal a obliteração mecânica dos túbulos dentinários 14, 15.

As restaurações em compósito se aderem à estrutura dentária por meio da associação dos sistemas adesivos, dando origem a uma interface denominada como camada híbrida. Atualmente, os sistemas adesivos universais são combinados com um primer acídico e bonding na mesma solução, sendo um produto versátil e de fácil utilização, já que o mesmo possui diversas possibilidades e técnicas, assim o clínico pode eleger qual técnica usar de acordo com cada caso 12, 17, 18. Diferentes estudos comprovaram que estes adesivos demonstraram bons resultados quanto à força de união em dentina, e isto tem sido atribuído aos monômeros acídicos incorporados na formulação química deste produto, que são capazes de promover uma ligação química de hidrocarbonetos de hidroxiapatita do dente. Estes adesivos podem ser aplicados em ambas as estratégias de adesão, tanto na Técnica Autocondicionante quanto na Técnica Condicione e Lave 13, 17, 18, assim como foi testado em nosso estudo.

A Técnica Condicione e Lave promove um condicionamento total da superfície, devido à aplicação separada do ácido fosfórico com subsequente lavagem e secagem da cavidade, para posteriormente realizar a hibridização do substrato. Em esmalte, este processo possibilita a formação de uma camada híbrida forte do ponto de vista mecânico. Entretanto, em dentina, esta técnica adesiva é mais crítica, já que cuidados relativos ao tempo do condicionamento ácido que promoverá a desmineralização dentinária e a consequente exposição das fibrilas de colágeno, bem como a quantidade ideal de umidade do substrato, deverão ser controlados. Isto torna o processo adesivo extremamente sensível à técnica, podendo gerar falhas adesivas em longo prazo, manchamento marginal e sensibilidade pós-operatória 17, 18, 19, 20. Em vista disso, a maior incidência da sensibilidade pós-operatória foi registrada no grupo com a realização do condicionamento ácido, presume-se que ocorreu devido à presença de uma dentina 
desmineralizada que não foi infiltrado pelos monômeros, no qual esta falha na camada híbrida pode acarretar em um desequilíbrio hidrodinâmico 4, 18.

Já a Técnica Autocondicionante não requer a realização dos passos de lavagem e secagem do preparo cavitário, uma vez que dispensa o condicionamento ácido prévio. Esta técnica depende, portanto, da desmineralização do tecido dentário por meio de um primer acídico associado ao adesivo, que acarretaria em uma hibridização teoricamente sem falhas, em razão da infiltração monomérica no substrato simultaneamente ao processo de desmineralização. Logo, diversos estudos atribuíram vantagens indiscutíveis a essa estratégia, como a diminuição da sensibilidade pós-operatória e a simplificação da técnica, em consequência da diminuição dos passos clínicos. Por outro lado, alguns autores evidenciam que a Técnica Autocondicionante demonstra baixos níveis de resistência de união no esmalte quando comparado com a Técnica Condicione e Lave, já outros autores rebatem mostrando resultados similares à técnica com condicionamento ácido prévio 17, 18, 19, 20.

$\mathrm{Na}$ literatura disponível, poucos trabalhos foram encontrados avaliando o sistema adesivo Ambar Universal. Um estudo evidenciou que o desempenho do Ambar Universal foi similar a todos os adesivos universais, não dependendo da estratégia adesiva utilizada 21. Entretanto, alguns autores apontam que o adesivo Ambar Universal apresenta uma menor resistência de união, quando utilizado nas estratégias autocondicionante e convencional em dentina seca 22, 23.

A avaliação clínica ainda é o melhor método para analisar a longevidade das restaurações em compósito, no entanto, ainda é pouco utilizada decorrente a exigência frente à dedicação dos pesquisadores, cooperação dos voluntários e os resultados que só são adquiridos após um tempo de proservação 25. Neste estudo, a sensibilidade foi provocada através de um estímulo tátil com uma sonda exploradora e um estímulo evaporativo com o jato de ar da seringa tríplice; trata-se de um método seguro de avaliação da intensidade de dor ante o estímulo, pois estes não geram agressões patológicas no complexo dentino-pulpar. A intensidade da sensibilidade dental foi medida através da escala visual analógica de dor, sendo considerado um método simples e de fácil interpretação, que pode ter alguns limites considerando a heterogeneidade de caráter pessoal da dor. No entanto, estudos anteriores argumentaram que esse método pode ser considerado adequadamente confiável 25. A amostra experimental seguiu fielmente os critérios de inclusão e exclusão com o objetivo de torná-la mais padronizada, e diminuir a ocorrência de algum viés que poderiam interferir nos índices de sensibilidade. Contudo, na literatura ainda há uma falta de evidência clínica cientifica abordando os sistemas adesivos universais e o tema citado. Portanto, mais estudos, principalmente clínicos de longevidade, são necessários para determinar a efetividade de sistemas adesivos universais em diferentes estratégias de adesão quanto ao tratamento da hipersensibilidade dentinaria em lesões cervicais não cariosas, porém, sua versatilidade de uso faz com que seja um produto de grande potencial de uso pelos clínicos em geral.

\section{CONCLUSÃO}

Com base nos resultados deste estudo, foi possível concluir que o tratamento restaurador com a utilização do adesivo Ambar Universal em ambas as estratégias de adesão produziu resultados semelhantes em relação à sensibilidade pós-operatória de sete dias. Como também, o tratamento restaurador com a utilização do adesivo Ambar Universal mostrou-se eficaz na redução da hipersensibilidade dentária em lesões cervicais não cariosas.

\section{AGRADECIMENTOS E FINANCIAMENTO}

Ao Comitê Institucional de Iniciação Científica da Universidade São Francisco e pelo apoio financeiro PROBAICITExt/USF.

\section{REFERÊNCIAS}

1. Teixeira DNR, Zeola LF, Machado AC, Gomes RR, Souza PG, Mendes DC, et al. Relationship Between Noncarious Cervical Lesions, Cervical Dentin Hypersensitivity, Gingival Recession, And Associated Risk Factors: A Cross-Sectional Study. Journal Of Dentistry. 2018;76:93-97.

DOI: $10.33947 / 1982-3282-v 15 n 3-4-4745$ 
2. Wierichs RJ, Kramer EJ, Meyer-Lueckel H. Risk Factors For Failure Of Class V Restorations Of Carious Cervical Lesions In General Dental Practices. Journal Of Dentistry. 2018;77:87-92.

3. Teixeira DNR, Thomas RZ, Soares PV, Cune MS, Gresnigt MMM, Slot DE. Prevalence Of Noncarious Cervical Lesions Among Adults: A Systematic Review. Journal Of Dentistry. 2020;95:103285.

4. Brännström M. Etiology Of Dentin Hypersensitivity. Proceedings of the Finnish Dental Society. 1992;88 Suppl 1:713.

5. Machado AC, Maximiano V, Eduardo CP, Azevedo LH, Freitas PM, Aranha AC. Associative Protocol For Dentin Hypersensitivity Using Nd:YAG Laser And Desensitizing Agent In Teeth With Molar-Incisor Hypomineralization. Journal Of Photobiomodulation, Photomedicine, And Laser Surgery. 2019;37(4):262-266.

6. Soares PV, Zeola LF, Cunha-Cruz J. Prevalence Of Dentin Hypersensitivity: Systematic Review And Meta-Analysis. Journal Of Dentistry. 2019;81:1-6.

7. Idon PI, Esan TA, Bamise CT, Mohammed ASA, Mohammed A, Ofuonye ILN. Dentine Hypersensitivity: Review Of A Common Oral Health Problem. Journal Of Dental And Craniofacial Research, 2017;2(2):16-23.

8. Giannini M, Ayres AP, Bonvent JJ, Mogilevych B, Soares LES, Martin AA, et al. Effect Of Non-Thermal Atmospheric Plasma On The Dentin-Surface Topography And Composition And On The Bond Strength Of A Universal Adhesive. European Journal Of Oral Sciences. 2018;126(1):53-65.

9. Nagarkar S, Theis-Mahon N, Perdigão J. Universal Dental Adhesives: Current Status, Laboratory Testing, And Clinical Performance. Journal Of Biomedical Materials Research Part B: Applied Biomaterials. 2019;107(6):2121-2131. 10. Silva EOS, Beltrani FC, Shibayama R, Contreras EFR, Hoeppner MG. Adhesive Systems: Concept, Aplication And Effectiveness. Arq. Ciênc. Saúde UNIPAR. 2010;14(1):81-87.

11. Ayer A. Association Between Severity Of Tooth Wear And Dentinal Hypersensitivity. Journal Of College Of Medical Sciences-Nepal. 2016;12(3):94-98.

12. Saikaew P, Fu J, Chowdhury AFMA, Carvalho RM, Sano H. Effect Of Air-Blowing Time And Long-Term Storage On Bond Strength Of Universal Adhesives To Dentin. Clinical Oral Investigations. 2019;23(6):2629-2635.

13. Chowdhury AFMA, Islam R, Alam A, Matsumoto M, Yamauti M, Carvalho RM, et al. Variable Smear Layer And Adhesive Application: The Pursuit Of Clinical Relevance In Bond Strength Testing. International Journal Of Molecular Sciences. 2019;20(21):5381.

14. Costa LS, Alves SSS, Lima DDC, Dietrich L, Santos-Filho PCF, Martins VM. Non-Carious Cervical Lesions And Dental Hypersensitivity: A Clinical Case Report. Revista Odontológica do Brasil Central. 2018;27(83):247-251.

15. Faria GJM, Villela LC. Etiology And Treatment Of The Dentinal Hypersensitivity In Teeth With Noncarious Cervical Lesions. Revista Biociências. 2000;6(1):21-27.

16. Trentin MS, Bervian J. Cervical Dentinal Hypersensitivity: A Review Of Literature. Revista da Faculdade de Odontologia - UPF. 2014;19(2):252-257.

17. Soares LP, Oliveira AC, Delvizio VC, Paes PNG, Lopes LS, Miranda MS. Aplicabilidades Clínicas Do Sistema Adesivo Universal: Relatos De Casos. Comunicação Científica E Técnica Em Odontologia - Atena Editora. 2020;4(1):1-11.

DOI: $10.33947 / 1982-3282-v 15 n 3-4-4745$ 
18. Pereira CD. Sistemas Adesivos Universais: Revisão Da Literatura. [Mestrado] Porto: Universidade Fernando Pessoa; 2019.

19. Giannini M, Makishi P, Ayres APA, Vermelho PM, Fronza BM, Nikaido T, et al. Self-Etch Adhesive Systems: A Literature Review. Brazilian Dental Journal. 2015;26(1):3-10.

20. Münchow EA. Síntese De Monômero Ácido Alternativo Para Composição De Sistemas Adesivos Autocondicionantes. []Dissertação para obtenção do título de Mestre em Odontologia]; Pelotas: Faculdade de Odontologia da Universidade Federal de Pelotas; 2014.

21. Siqueira FS, Cardenas AM, Ocampo JB, Hass V, Bandeca MC, Gomes JC, et al. Bonding performance of universal adhesives to eroded dentin. The Journal of Adhesive Dentistry. 2018;20(2):121-132.

22. Dutra JBD. Avaliação da resistência de união de sistemas adesivos universais à dentina humana, utilizando diferentes estratégias de adesão. [Dissertação]. Belo Horizonte; 2018.

23. Cardoso GC, Nakanishi L, Isolan CP, Jardim PD, Moraes RR. Bond Stability of Universal Adhesives applied to dentin using Etch-And-Rinse or Self-Etch Strategies. Brazilian Dental Journal. 2019;30(5):467-475.

24. Peumans M, De Munck J, Van Landuyt KL, Poitevin A, Lambrechts P, Van Meerbeek B. Eight-year clinical evaluation of a 2-step self-etch adhesive with and without selective enamel etching. Dental Materials. 2010;26:1176-1184. 25. Balto K. Single- or multiple-visit endodontics: which technique results in fewest postoperative problems?. Evidence-Based Dentistry. 2009;10(1):16. 